\title{
First investigation of excited states in the odd-proton nucleus ${ }^{209} \mathrm{Fr}$
}

\author{
D. A. Meyer, ${ }^{1}$ C. W. Beausang, ${ }^{1,2}$ J. J. Ressler, ${ }^{1,3}$ H. Ai,${ }^{1}$ H. Amro, ${ }^{1}$ M. Babilon,${ }^{1,4}$ R. F. Casten, ${ }^{1}$ C. R. Fitzpatrick, ${ }^{1,5}$ \\ G. Gürdal, ${ }^{1,6}$ A. Heinz, ${ }^{1}$ E. A. McCutchan, ${ }^{1}$ C. Plettner, ${ }^{1}$ J. Qian, ${ }^{1}$ N. J. Thomas, ${ }^{1,5}$ V. Werner, ${ }^{1}$ \\ E. Williams, ${ }^{1}$ N. V. Zamfir, ${ }^{1,7}$ and Jing-ye Zhang ${ }^{8}$ \\ ${ }^{1}$ Wright Nuclear Structure Laboratory, Yale University, New Haven, Connecticut 06520-8124, USA \\ ${ }^{2}$ Physics Department, University of Richmond, Richmond, Virginia 23173, USA \\ ${ }^{3}$ Department of Chemistry, Simon Fraser University, Burnaby, British Columbia, Canada V5A-1S6 \\ ${ }^{4}$ Institut für Kernphysik, Technische Universität Darmstadt, D-64289 Darmstadt, Germany \\ ${ }^{5}$ University of Surrey, Guildford, Surrey GU2 7XH, United Kingdom \\ ${ }^{6}$ Clark University, Worcester, Massachusetts 01610, USA \\ ${ }^{7}$ National Institute of Physics and Nuclear Engineering, Bucharest, Romania \\ ${ }^{8}$ Department of Physics and Astronomy, University of Tennessee, Knoxville, Tennessee 37996, USA
}

(Received 27 September 2005; published 8 February 2006)

\begin{abstract}
Excited states in ${ }^{209} \mathrm{Fr}$ were produced following the ${ }^{176} \mathrm{Yb}\left({ }^{37} \mathrm{Cl}, 4 n\right)$ reaction. An excitation function was measured with data taken at 173, 179, and $185 \mathrm{MeV}$. Recoiling fusion-evaporation products were separated using the SASSYER gas-filled spectrometer. HPGe clover detectors of the YRAST Ball array at the target position and at the focal plane were used to detect prompt and delayed gamma ray decays, providing the first spectroscopic information about prompt and delayed transitions in ${ }^{209} \mathrm{Fr}$. The decay from a proposed $\pi i_{13 / 2}$ configuration isomer was observed in ${ }^{209} \mathrm{Fr}$, and its half-life was measured to be $446(14) \mathrm{ns}$. States above the isomer were also observed.
\end{abstract}

DOI: 10.1103/PhysRevC.73.024307 PACS number(s): 23.20.Lv, 21.10.Tg, 25.70.Gh, 27.80.+w

\section{INTRODUCTION}

A wide variety of structural phenomena have been predicted and observed in the region of the nuclear chart near doublymagic ${ }^{208} \mathrm{~Pb}$ in nuclei with $N \leqslant 126$. These nuclei can be populated via fusion evaporation reactions and studied using gamma ray spectroscopic methods. Their structural features include shears bands, superdeformation, and shape coexistence, among others (for example, Refs. [1-6]). Coupling of single-particle states to the $\mathrm{Pb}$ core is important in these nuclei because of nearby magic numbers $N=126$ and $Z=82$.

A considerable amount of spectroscopic information is available for isotopes with $Z \leqslant 82$ [7-20]; however, for nuclei with $Z>82$, the situation is less satisfying. The structure of even-even Po, Rn, and, to a lesser extent, Ra nuclei have been well-established, but relatively less spectroscopic information is available for odd- $Z$ isotopes. Even less spectroscopic information is available for doubly odd nuclei. One reason for this dearth of experimental information is the rapidly increasing fissility. To overcome this difficulty, careful experimental selection of fusion-evaporation residues becomes critical. In addition, for odd and doubly odd nuclei, single particle states with different proton and neutron configurations occur at similar energies and can be difficult to resolve. Thus, in addition to the large fission competition (and reduced fusion evaporation cross section) the level schemes of these nuclei are generally more complicated than their even-even counterparts. However, study of the odd isotopes provides useful information about the degrees of freedom of the odd particle and thus can give direct insights into the structure and deformation of these nuclei and the mechanisms that affect the evolution of structure and shape.

Prior to this work, spectroscopic information for Fr isotopes below the closed neutron shell at $N=126$ was very limited, and was available only for ${ }^{213} \operatorname{Fr}(N=126),{ }^{212} \operatorname{Fr}(N=125)$, and ${ }^{211} \operatorname{Fr}(N=124)$ [21]. Excited states in these nuclei have been interpreted in terms of single particle configurations of a few valence particles outside the $N=126$ closed shell. (Information for ${ }^{210} \mathrm{Fr}$, populated via alpha decay of ${ }^{214} \mathrm{Ac}$, also exists [22].) In an effort to extend the scope of spectroscopic information in this region to lighter systems, decays above and below a new isomeric state in ${ }^{209} \mathrm{Fr}$ are reported here for the first time.

\section{EXPERIMENT}

Experiments to populate excited states in ${ }^{209} \mathrm{Fr}$ were performed at the Wright Nuclear Structure Laboratory at Yale University. In the first, a beam of ${ }^{37} \mathrm{Cl}$ ions incident on a thin $\left(1 \mathrm{mg} / \mathrm{cm}^{2}\right){ }^{176} \mathrm{Yb}$ target produced $\mathrm{Fr}$ and $\mathrm{Rn}$ isotopes via the $\left({ }^{37} \mathrm{Cl}, x n \gamma\right)$ and $\left({ }^{37} \mathrm{Cl}\right.$, pxn $\left.\gamma\right)$ reaction channels, respectively, at beam energies of 173,179 , and $185 \mathrm{MeV}$. An array of six Compton-suppressed clover HPGe detectors, each with $\sim 150 \%$ relative efficiency, detected prompt gamma rays emitted at the target position. These detectors were situated in two rings of the YRAST Ball array [23] with two detectors at $90^{\circ}$ to the beam axis and four detectors at $138.5^{\circ}$ to the beam axis. The overall efficiency of the array in this configuration is $\sim 1.8 \%$ at $1.3 \mathrm{MeV}$.

Following the fusion evaporation reaction, recoiling fusion products were selected using the Small Angle Separator System at Yale for Evaporation Residues (SASSYER, Ref. [24]). The spectrometer was filled with 0.8(1) Torr He gas, contained by a thin $\left(50 \mathrm{mg} / \mathrm{cm}^{2}\right) \mathrm{C}$ foil placed $1.5 \mathrm{~m}$ upstream of the target chamber. The beam lost nearly $0.8 \mathrm{MeV}$ traversing the carbon window. SASSYER was set to select $A=209$ recoils with a magnetic rigidity of $1.9 \mathrm{Tm}$. Fission 


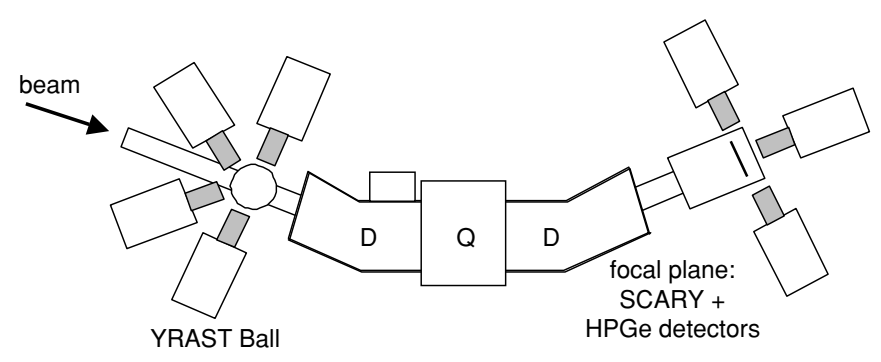

FIG. 1. The beam is incident (from the left of this figure) on a target centered at YRAST Ball. The recoils are then focused to the focal plane by setting the fields of two dipole magnets and one quadrupole magnet. Recoiling nuclei are detected in SCARY, an array of 30 solar cells. Prompt gamma rays are detected using YRAST Ball, and delayed gamma rays are detected using Ge detectors around the focal plane.

fragments and unreacted beam particles, well separated from fusion products, were bent into a beam dump box located at the first dipole of SASSYER.

After a $\sim 2.4 \mathrm{~m}$ flight path, recoils were implanted into a solar cell array consisting of $30 \mathrm{Si}$ solar cells located at the focal plane of SASSYER. Mass $A \sim 209$ recoils, traveling at a speed of $0.0155 \mathrm{c}$ (as experimentally measured for $A=209$ recoils using the Doppler shift observed in clover detectors at the target position) have a time of flight through SASSYER of $\sim 500$ ns from production to implantation at the focal plane. The solar cell array was $3 \mathrm{~cm}$ high by $10 \mathrm{~cm}$ wide; each cell covered an area of $1 \mathrm{~cm}^{2}$. This array gave the position and energy of the recoils and generated the master trigger (start) for each event. Since the area covered by each element of the solar cell array is too large for recoil-decay-tagging, simple recoil-tagging was used to correlate implantation with either prior gamma decay at the target position or subsequent gamma decay from isomeric states at the focal plane.

To detect such gamma rays emitted from the decay of isomeric states, three clover detectors were placed in close proximity to the focal plane chamber. Two of these were placed facing the sides of the solar cell array, while the third was placed directly behind the array. The experiment was sensitive to lifetimes on the order of $\sim 10$ ns measured at the target position and from about $300 \mathrm{~ns}$ to $8 \mu \mathrm{s}$ at the focal plane. A schematic diagram of the experimental setup is shown in Fig. 1.

For the 173, 179, and $185 \mathrm{MeV}$ beam energies, 40, 12, and $98 \mathrm{~h}$ of data, respectively, were taken with an average beam current of $I_{b} \sim 4 \mathrm{pnA}$. At a beam energy of $185 \mathrm{MeV}$, a total of $7.5 \times 10^{6}$ recoils, $2.5 \times 10^{6}$ recoil-gamma, $0.8 \times 10^{6}$ recoilgamma-gamma, $9.7 \times 10^{4}$ recoil-isomer, $3.0 \times 10^{4}$ recoilisomer-isomer, and $7.7 \times 10^{4}$ recoil-gamma-isomer unfolded coincidence events were recorded. For the last $53 \mathrm{~h}$ of data taken at $185 \mathrm{MeV}$, approximately half of the time spent at this beam energy, the master trigger condition was changed to record data either when an event occurred in the solar cell array or when the prompt gamma ray multiplicity was greater than or equal to 3 .

A second experiment was performed using only the Compton suppressed HPGe detectors of YRAST Ball at the target position of SASSYER. For this experiment, ${ }^{16} \mathrm{O}$ ions at $90 \mathrm{MeV}$ were incident on a thick $\left(4 \mathrm{mg} / \mathrm{cm}^{2}\right){ }^{197} \mathrm{Au}$ target. The target was sufficiently thick to stop recoiling fusion evaporation products, and the beam energy was chosen to match the excitation energy of the fusion products produced in the thin target experiment. This beam energy was also chosen to maximize the yield of Fr evaporation residues relative to the fission channel [25]. The beam lost $\sim 6 \mathrm{MeV}$ in the target [26], and ${ }^{210} \mathrm{Fr}$ was primarily populated. Using a coincidence requirement of three or more suppressed clover detectors, approximately $8 \times 10^{7}$ unfolded gamma-gamma events were collected over a period of $29 \mathrm{~h}$.

\section{RESULTS}

No spectroscopic information for excited states in Fr nuclei with $N<124$ (lighter than ${ }^{211} \mathrm{Fr}$ ) was available prior to these experiments. Therefore, gamma rays were assigned to Fr based on coincidences with Fr X-rays and to specific isotopes based on the results of our excitation function measurement using the thin target data collected at three beam energies $(173,179$, and $185 \mathrm{MeV}$ ) and on gamma-gamma coincidences. For the

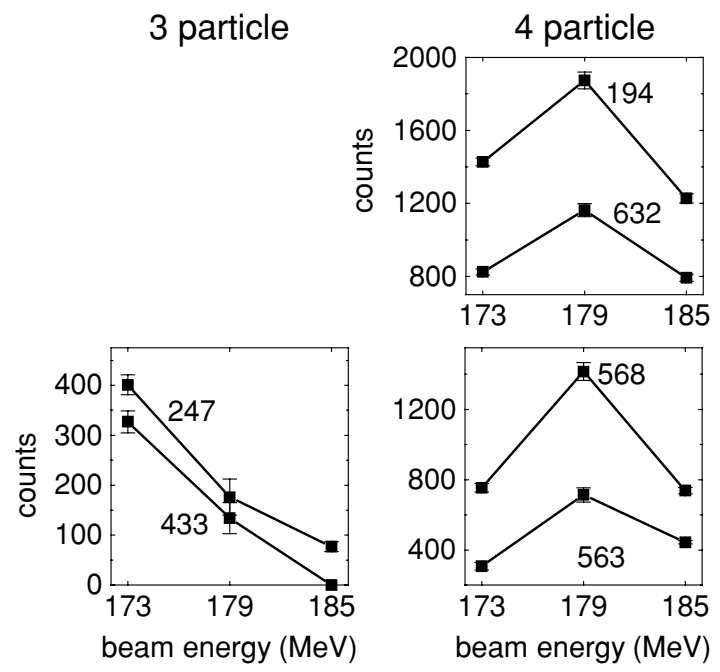

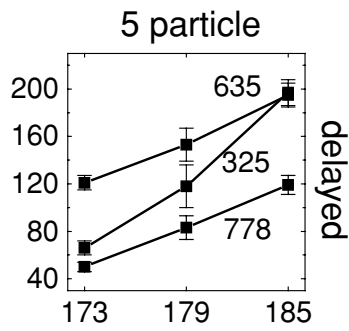

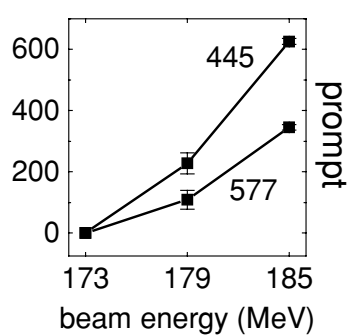

024307-2
FIG. 2. Excitation function measured in this work for the 3, 4, and 5 particle channels. Top panels show gamma rays detected at the focal plane. Bottom panels show gamma rays detected at the target position. The 194, 632, 568, and $563 \mathrm{keV}$ transitions belong to ${ }^{209} \mathrm{Fr}$. The 635 , 325,778 , and $577 \mathrm{keV}$ transitions belong to ${ }^{208} \mathrm{Rn}$. For details, see text. (Note the suppressed zero in some of the plots.) 

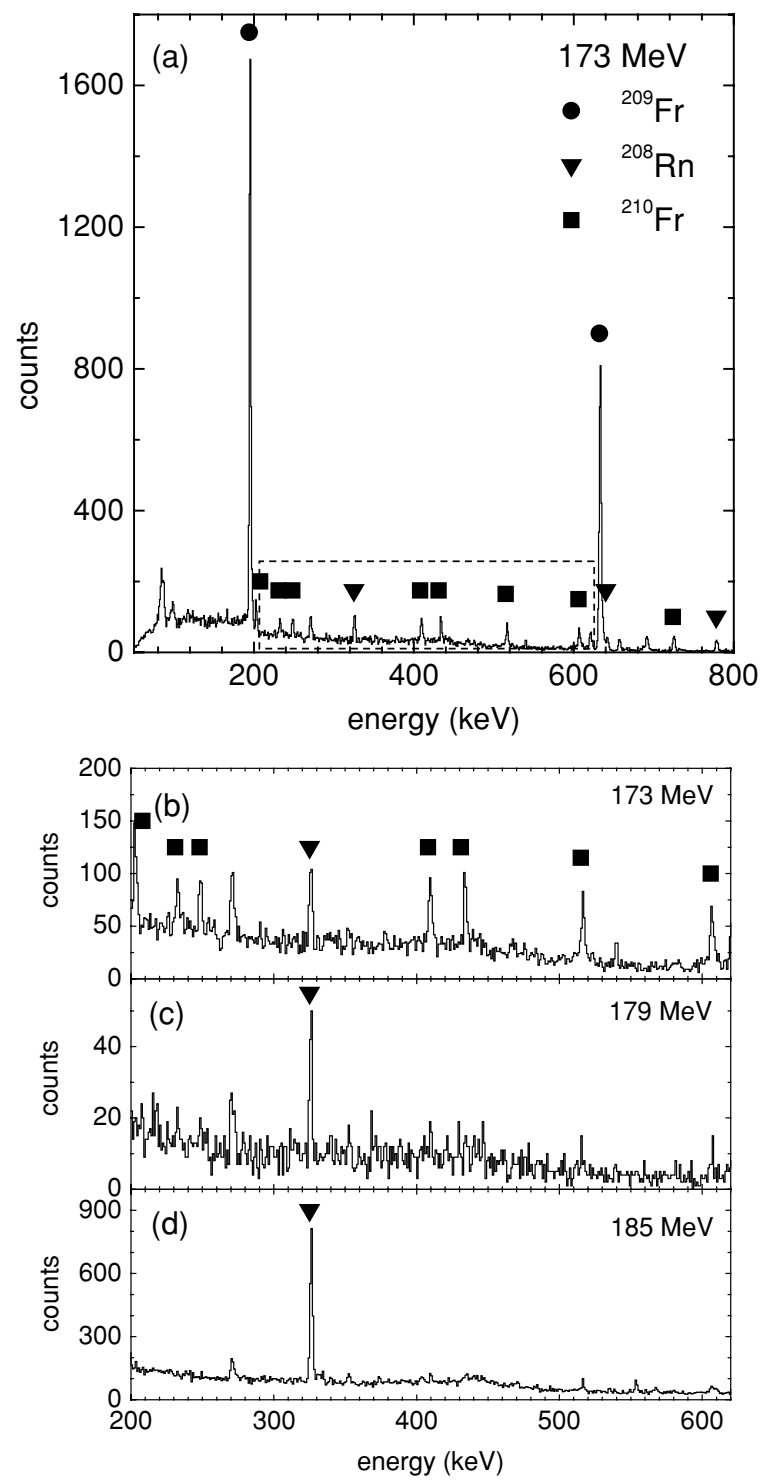

FIG. 3. Gamma ray spectra obtained at the focal plane at 173, 179, and $185 \mathrm{MeV}$. Top: Portion of spectrum (a) of delayed gamma rays measured at the focal plane at a beam energy of $173 \mathrm{MeV}$. The spectrum is dominated by the 194 and $632 \mathrm{keV}$ gamma rays belonging to ${ }^{209} \mathrm{Fr}$. Bottom: Portion of the spectrum indicated by dashed box in (a) between the 194 and $632 \mathrm{keV}$ gamma rays at (b) 173, (c) 179, and (d) $185 \mathrm{MeV}$ beam energies. The varying strengths of the 3, 4, and 5 particle channels are evident from the relative intensities of the corresponding gamma rays.

excitation function measurement, the variation, as a function of beam energy, of the intensities of both prompt and delayed (measured at the focal plane of SASSYER) gamma rays was observed. To account for the different beam fluxes at the three beam energies, the gamma ray intensities were normalized to the $188 \mathrm{keV} 4^{+}$to $2^{+}$transition in the Coulomb-excited ${ }^{176} \mathrm{Yb}$ target. In the energy range from $173-185 \mathrm{MeV}$, the total Coulomb-excitation cross section, calculated according to Ref. [27], for this state is essentially constant. The resulting excitation function measurement is shown in Fig. 2. As can be seen, known transitions in ${ }^{208} \mathrm{Rn}$, populated following the $p 4 n$ reaction, exhibit an upward trend with increasing beam energy, establishing the excitation function of the five-particle evaporation channel. In contrast, previously unknown and unplaced transitions from Fr isotopes constitute the majority of the gamma rays following downward (three particle) and peaked (four particle) trends.

Delayed gamma rays emitted within $8 \mu$ s of recoil implantation were detected at the focal plane. While the statistics are somewhat poorer, the focal plane spectra are extremely clean, making unambiguous measurements possible. The spectrum of gamma rays detected following a recoil implantation is shown in Fig. 3. As can be seen, it is dominated by the 194 and $632 \mathrm{keV}$ transitions. In addition to these gamma-rays, the known 325, 636, $778 \mathrm{keV}$ transitions, below the $487 \mathrm{~ns}$ $8^{+}$isomer in ${ }^{208} \mathrm{Rn}$ [15], are seen as well as many other less intense, previously unplaced transitions. As demonstrated by the known transitions in ${ }^{208} \mathrm{Rn}$, the shape of the excitation function for gamma rays detected at the focal plane mirrors that of the excitation function for prompt gamma rays. Thus, the excitation function, measured either at the target position or at the SASSYER focal plane, can be used to assign transitions to specific nuclei.

The excitation function for the 194 and $632 \mathrm{keV}$ gamma rays indicates that they belong to the four-particle channel. They are mutually coincident and also coincident with the $86 \mathrm{keV}$ Fr x-ray detected at the focal plane. In addition, these delayed transitions are correlated with prompt 86 and $97 \mathrm{keV}$ Fr x-rays detected at the target position. The combination of excitation function and $\mathrm{x}$-ray coincidence data unambiguously place the 194 and $632 \mathrm{keV}$ transitions in ${ }^{209} \mathrm{Fr}$.

The half-lives of the states depopulated by these transitions can be determined from a weighted fit to the time difference between recoil implantation and gamma decay for each transition. Using this method, we obtain a half-life of 482(20) ns for the isomer in ${ }^{208} \mathrm{Rn}$ (325 keV transition, see Fig. 4) which is

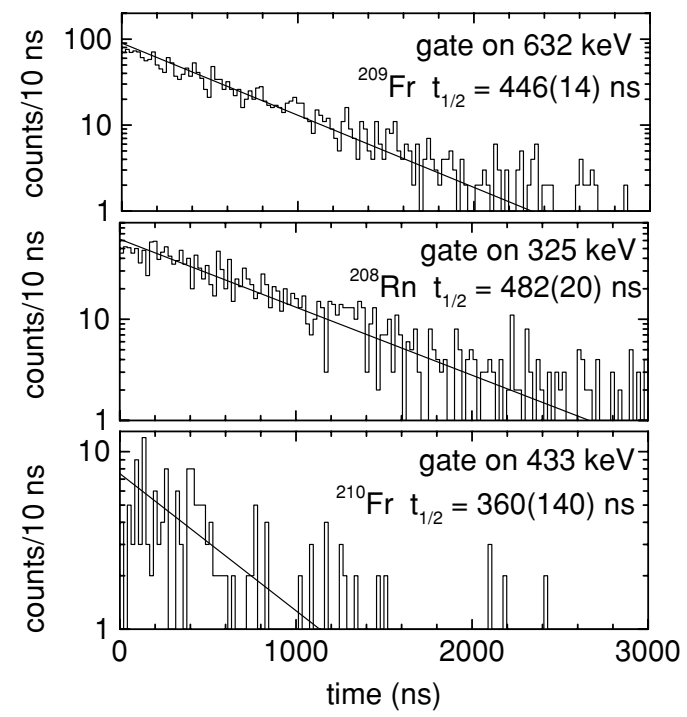

FIG. 4. Time relative to implantation for several gamma rays measured at the focal plane. The solid line shows a weighted least squares fit to decay spectrum of isomeric gamma rays: $632 \mathrm{keV}$ $\left({ }^{209} \mathrm{Fr}\right), 325 \mathrm{keV}\left({ }^{208} \mathrm{Rn}\right)$, and $433 \mathrm{keV}\left({ }^{210} \mathrm{Fr}\right)$. 
in excellent agreement with the previously measured value of 487(12) ns [15]. Similar fits for the 194 and $632 \mathrm{keV}$ transitions show that they have the same half-life, indicating that both transitions follow from the decay of the same isomeric state in ${ }^{209} \mathrm{Fr}$. The half-life of this new isomer is measured to be 446(14) ns; the decay curve for the $632 \mathrm{keV}$ transition is shown in Fig. 4.

As can be seen in Fig. 3, the intensity of many of the other isomeric transitions is greatest at the lowest beam energy $(173 \mathrm{MeV})$, suggestive of a $3 n$ evaporation channel and an assignment to ${ }^{210} \mathrm{Fr}$. The coincidence data at the focal plane for these transitions have very low statistics and makes assignment to any isotope challenging. However, placement of these transitions in ${ }^{210} \mathrm{Fr}$ was confirmed by the thick target experiment in which the same transitions were strongly populated. In this experiment, a $90 \mathrm{MeV}{ }^{16} \mathrm{O}$ beam incident on a $4 \mathrm{mg} / \mathrm{cm}^{2}{ }^{197} \mathrm{Au}$ target strongly populated ${ }^{210} \mathrm{Fr}$ via the $3 n$ channel. Here the assignment to Fr (based on $\mathrm{x}$-ray coincidences) is unambiguous, and the assignment to ${ }^{210} \mathrm{Fr}$ is based on the thin target excitation function and the relative excitation energy of the two compound systems. It was found that many of the transitions, observed at the focal plane of SASSYER in the thin target experiment, are mutually coincident using the thick target data. Hence, we can deduce that there is at least one long-lived high spin isomer in ${ }^{210} \mathrm{Fr}$. Although the statistics at the focal plane are very poor for these transitions, a similar weighted fit to the half-life of the $433 \mathrm{keV}$ state yields a 360(140) ns half-life, shown in Fig. 4. The intensities for all of the delayed transitions are listed in Table I, where they have been corrected for relative efficiency under the approximation that radiation from the extended solar cell array is similar to ${ }^{152} \mathrm{Eu}$ and ${ }^{133} \mathrm{Ba}$ point sources placed near the center of the solar cell array. For the ${ }^{210} \mathrm{Fr}$ transitions, only the isotope assignment is given here; detailed results for this nucleus will be presented in a subsequent publication [28].

As for the delayed transitions, prompt transitions tagged by recoils in the solar cell array were assigned to a specific element based on x-ray coincidences and to ${ }^{209} \mathrm{Fr}$ by the excitation function results and by coincidences with delayed gamma-rays detected at the focal plane. For example, prompt transitions coincident with the 194 and $632 \mathrm{keV}$ delayed transitions are shown in Fig. 5. These are assigned as higher

TABLE I. Energies and relative gamma ray intensities for transitions in ${ }^{210} \mathrm{Fr}$ as detected at the focal plane of SASSYER. They lie below at least one isomeric level (see Fig. 4). These intensities, normalized to the $433 \mathrm{keV}$ transition, have been corrected for efficiency but not for internal conversion.

\begin{tabular}{lcc}
\hline \hline Nucleus & Energy (keV) & Relative intensity \\
\hline${ }^{210} \mathrm{Fr}$ & $202.1(4)$ & $0.5(1)$ \\
& $231.4(4)$ & $0.4(1)$ \\
& $247.4(4)$ & $0.6(1)$ \\
& $408.8(4)$ & $1.1(1)$ \\
& $433.5(4)$ & $1.0(1)$ \\
& $515.2(4)$ & $1.1(2)$ \\
& $619.5(5)$ & $1.1(3)$ \\
& $690.9(4)$ & $1.3(1)$ \\
\hline \hline
\end{tabular}
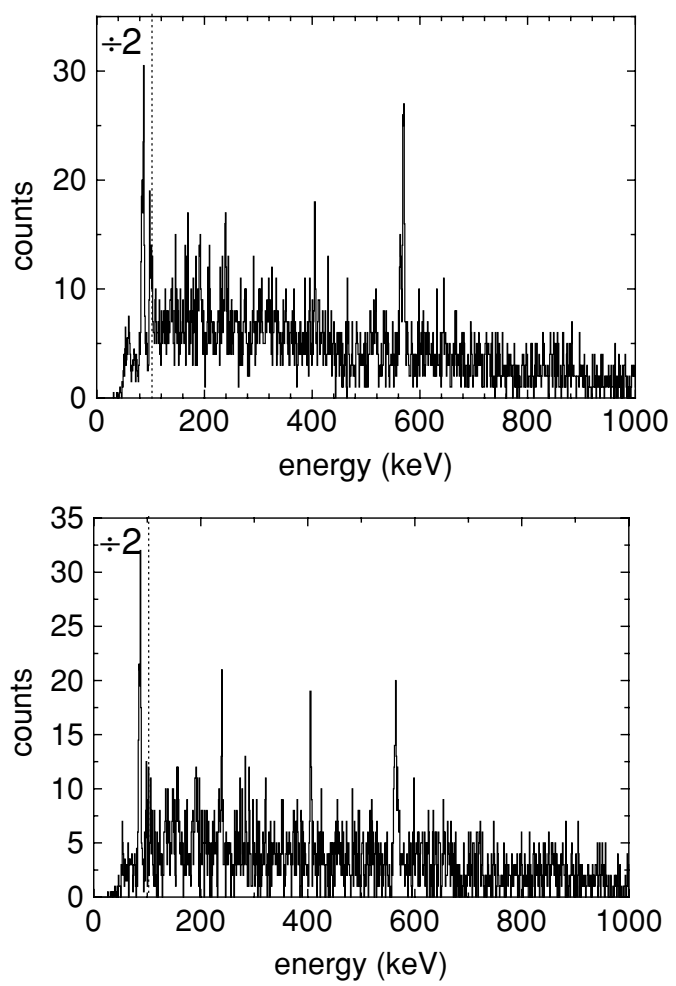

FIG. 5. Gate on $632 \mathrm{keV}$ isomeric transition (top) shows prompt gamma rays in coincidence. Gates in the recoil-gamma-gamma matrix, such as the gate on the $568 \mathrm{keV}$ transition (bottom), have been used for building the level scheme.

spin states feeding the $446 \mathrm{~ns}$ isomer in ${ }^{209} \mathrm{Fr}$. Using recoilgamma-gamma events, the level scheme shown in Fig. 6 was developed. Interestingly, the $632 \mathrm{keV}$ transition is also observed in the spectrum at the target position, and a gate on this transition yields two coincident transitions, 724 and $359 \mathrm{keV}$.

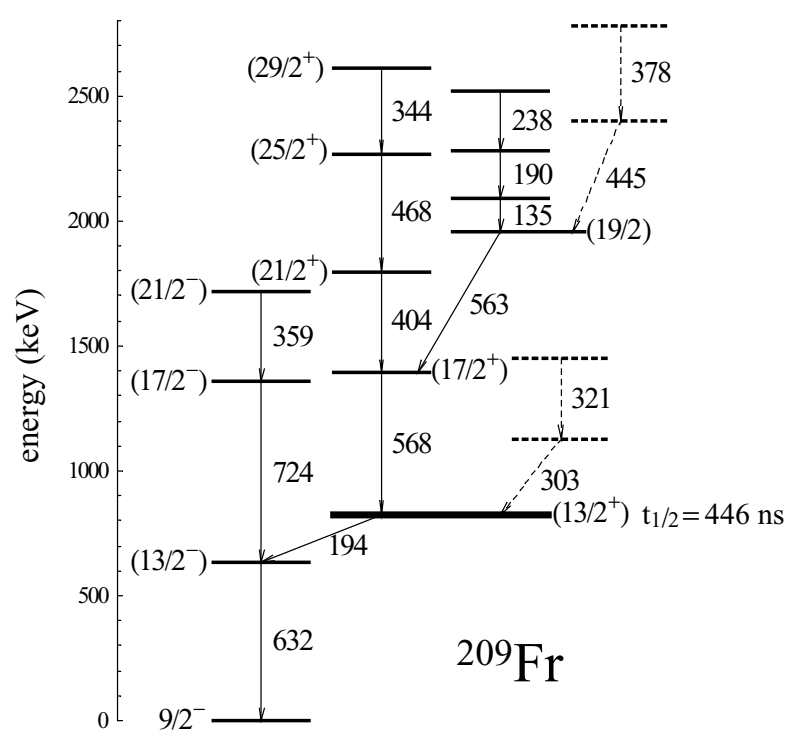

FIG. 6. Level scheme for ${ }^{209} \mathrm{Fr}$ obtained in this work. Dashed lines indicate tentative assignments. 
Some information on the electromagnetic nature of the gamma ray transitions can be determined by comparing the relative gamma ray intensities in the two YRAST Ball rings at $90^{\circ}$ and $138.5^{\circ}$. This information is limited both by the angular range of YRAST Ball at SASSYER and by the poor statistics. Nevertheless, tentative spin assignments can be made by assuming that for stretched transitions, quadrupole transitions are more intense in the backward $\left(138.5^{\circ}\right)$ ring, while dipole transitions are more intense in the ring at $90^{\circ}$. Tentative spins and parities for the levels are assigned using this information as well as by comparison to the systematic trends of neighboring isotopes. The energies and intensities of transitions observed in the prompt gamma ray spectrum are shown in Table II. The statistics at the focal plane are sufficient to allow reliable coincidence data for the 632 and $194 \mathrm{keV}$ transitions. A gate placed on the $194 \mathrm{keV}$ transition brings back only the $632 \mathrm{keV}$ transition. A gate on the 632 transition brings back the $194 \mathrm{keV}$ transition and Fr x-rays as would be expected from the substantial internal conversion of such a low energy transition. Since the 632 and $194 \mathrm{keV}$ transitions are fed from the same isomeric level and these nuclei have been selected using SASSYER, there is no possibility for side feeding. Therefore, the intensities of the two transitions at the focal plane must be equal. This intensity balance argument was used to deduce the internal conversion coefficient, $\alpha=0.5(2)$, for the $194 \mathrm{keV}$ transition, which is consistent with either an $E 1$ or $E 2$ assignment.

TABLE II. Energies and relative gamma ray intensities for transitions in ${ }^{209} \mathrm{Fr}$, measured in the total projections, as detected at the focal plane (delayed) and target position (prompt) of SASSYER. The intensities of the delayed transitions are normalized to the $632 \mathrm{keV}$ transition. The intensities of the prompt transitions are normalized to the $568 \mathrm{keV}$ transition. Transitions detected in both places have been corrected for their respective efficiencies. The internal conversion coefficient $\alpha$ and electromagnetic properties are discussed in the text.

\begin{tabular}{lccc}
\hline \hline & Energy (keV) & Relative intensity & $\alpha$ \\
\hline delayed & $194.1(4)$ & $0.57(1)$ & $0.5(3)$ \\
& $632.0(4)$ & $1.00(2)$ & \\
prompt & $134.9(7)$ & $0.06(1)$ & \\
$189.8(6)$ & $0.19(1)$ & \\
$237.8(5)$ & $0.26(1)$ & \\
$302.6(5)$ & $0.32(1)$ & \\
$321.3(5)$ & $0.25(1)$ & \\
& $343.8(5)$ & $0.05(2)$ & \\
$358.8(6)$ & $0.09(1)$ & \\
$378.0(5)$ & $0.13(1)$ & \\
$404.2(5)^{\mathrm{a}}$ & $0.38(1)$ & \\
& $444.9(6)$ & $0.41(1)$ & \\
& $468.1(5)$ & $0.04(1)$ & \\
& $563.2(5)^{\mathrm{b}}$ & $0.60(1)$ & \\
$568.1(5)^{\mathrm{a}}$ & $1.00(2)$ & \\
& $631.8(6)$ & $0.35(2)$ & \\
$724.4(5)$ & $0.29(3)$ & \\
\hline
\end{tabular}

${ }^{\mathrm{a} Q u a d r u p o l e . ~}$

${ }^{\mathrm{b}}$ Dipole.

\section{DISCUSSION}

The deformation for ${ }^{209} \mathrm{Fr}$, with $Z=87$ and $N=122$ and lying close the the doubly magic ${ }^{208} \mathrm{~Pb}$, is expected to be small. This is expectation is reinforced by ultimate cranker calculations (Fig. 7, Ref. [29]) that predict an equilibrium deformation corresponding to an oblate shape, with a small $\epsilon_{2} \sim 0.05$. This shape is more or less independent of the single particle configuration or signature. Single particle excitations will play a major role in the structure of ${ }^{209} \mathrm{Fr}$ as they do in neighboring nuclei. For this deformation, the relevant single particle proton orbits are $\pi 1 h_{9 / 2}, \pi 2 f_{7 / 2}$, and $\pi 1 i_{13 / 2}$ while the relevant high- $j$ neutron orbital is $v 1 h_{9 / 2}$.

States built upon these orbitals feature prominently in the neighboring odd proton $\mathrm{Bi}(Z=83)$ and $\mathrm{At}(Z=85)$ nuclei. The ground states are typically based on the $\pi h_{9 / 2}$ orbital. Excited states in the odd-Bi $[12,14,16]$ isotopes, plotted in Fig. 8, show the trends in excitation energy of the different single proton orbitals. As can be seen, the $f_{7 / 2}$ orbital lies at an excitation energy of $\sim 700-800 \mathrm{keV}$, and the $i_{13 / 2}$ orbital lies at a higher energy of $\sim 1600 \mathrm{keV}$. These energies remain relatively constant with decreasing neutron number (down to $N=120)$.

In the odd-At $(Z=85$, Refs. $[14,16,18])$ isotopes, these single particle states are less easily picked out of the existing level schemes. However, some indication of their excitation energy can be inferred by looking at the energies of states of the maximally aligned $23 / 2^{-} \pi f_{7 / 2} \otimes\left(\pi h_{9 / 2}\right)^{2}$ and $29 / 2^{+} \pi i_{13 / 2} \otimes\left(\pi h_{9 / 2}\right)^{2}$ configurations. Although not all of these states have been identified for these couplings, the general trends as a function of neutron number are shown in Fig. 8. While the excitation energies of the probable

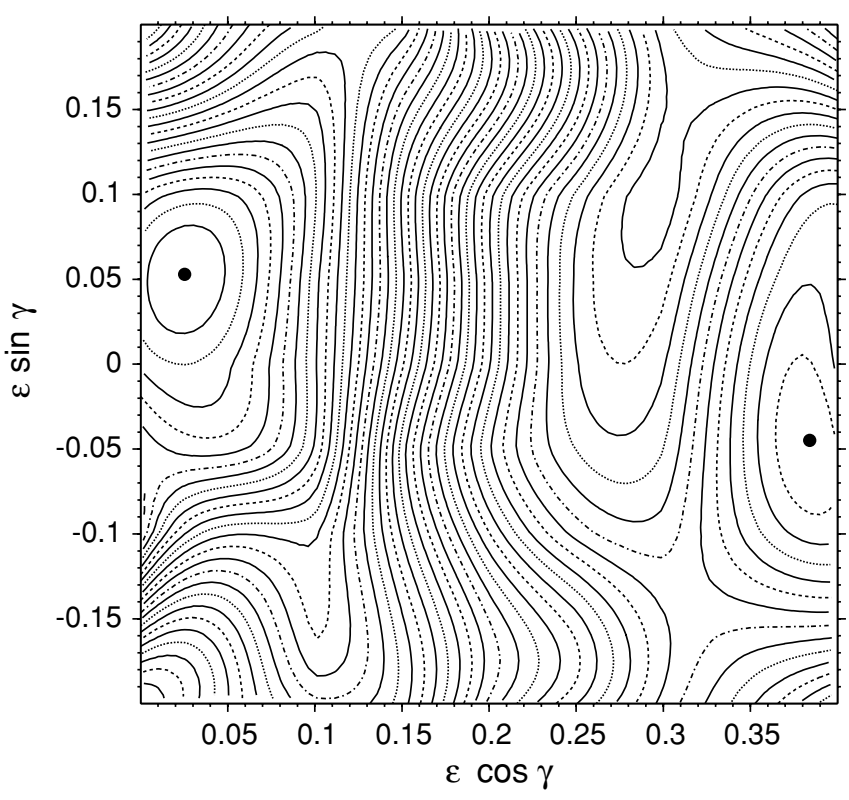

FIG. 7. Calculations done using the ultimate cranker model [29] with $\hbar \omega=0.23 \mathrm{MeV}$ for positive parity proton and neutron excitations, shown with contours of $200 \mathrm{keV}$. They show two minima at low excitation energies: One very deep, slightly oblate minimum (left) and one higher-lying, strongly prolate minimum (right). 


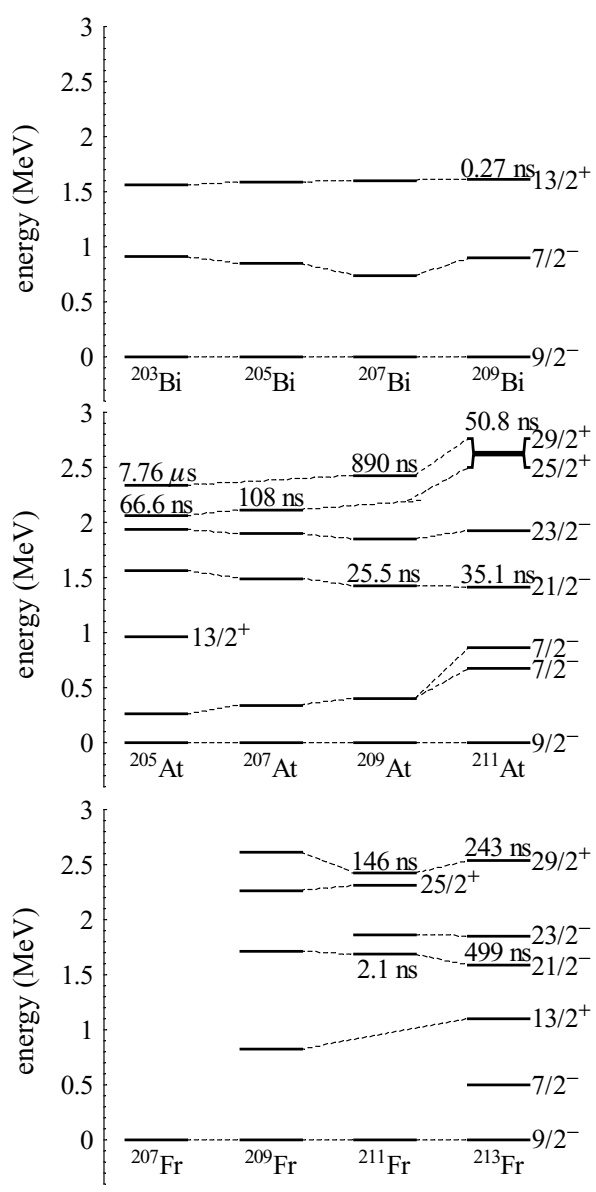

FIG. 8. Levels $[12,14,16,18,21]$ in the odd $\mathrm{Bi}$, At, and Fr isotopes indicative of the relative energies of the orbitals. The $29 / 2^{+}$and $25 / 2^{+}$ states are likely members of the $\pi i_{13 / 2} \otimes \pi\left(h_{9 / 2}\right)^{2}$ configuration. The $23 / 2^{-}$and $21 / 2^{-}$maximally aligned states presumably have the $\pi f_{7 / 2} \otimes \pi\left(h_{9 / 2}\right)^{2}$ and $\pi\left(h_{9 / 2}\right)^{3}$ configurations, respectively.

$23 / 2^{-} \pi f_{7 / 2} \otimes\left(\pi h_{9 / 2}\right)^{2}$ states remains relatively constant, the $29 / 2^{+}$and $25 / 2^{+} \pi i_{13 / 2} \otimes\left(\pi h_{9 / 2}\right)_{6}^{2}$ states appear to come down in energy. The $25 / 2^{+}$state approaches the excitation energy of the $23 / 2^{-}\left[\pi f_{7 / 2} \otimes\left(\pi h_{9 / 2}\right)^{2}\right]$ state at $N=120$. The relative spacing between what are likely the $25 / 2^{+}\left(\pi i_{13 / 2} \otimes\left[\left(\pi h_{9 / 2}\right)^{2}\right]_{6}\right)$ and $23 / 2^{-}$states is also important. In the odd proton nuclei where the states are known and where the $25 / 2^{+}$lies above the $23 / 2^{-}\left({ }^{211} \mathrm{At},{ }^{205} \mathrm{At}\right.$, and ${ }^{211} \mathrm{Fr}$ ), the typical decay path is from the maximally aligned $29 / 2^{+}$state via the $25 / 2^{+}$member of the same multiplet to the $23 / 2^{-}$and thence to the $21 / 2^{-}$maximally aligned states. From the $21 / 2^{-} \pi\left(h_{9 / 2}\right)^{3}$ state the decay path proceeds to the $h_{9 / 2}$ ground state via a cascade of three stretched $E 2$ transitions. Often the maximally aligned states are isomeric. As the $25 / 2^{+}$ state comes down in energy, the relative spacing between it and the $23 / 2^{-}$state becomes smaller, and the decay between them is somewhat inhibited. Thus, it appears that with increasing numbers of valence particles, the $i_{13 / 2}$ orbit comes down in energy relative to the $f_{7 / 2}$ orbit.

With the addition of two more valence protons in the odd-Fr isotopes, we would expect a similar, if not stronger, lowering of the energy of the $i_{13 / 2}$ orbital. For example, the relative spacing

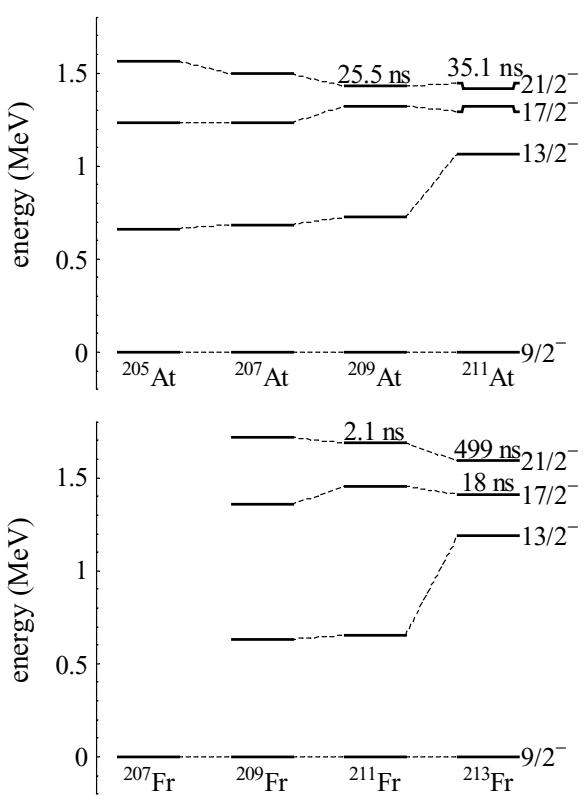

FIG. 9. Comparison of level spacings of the $\left(h_{9 / 2}\right)^{3}$ configurations in the odd-At $[14,16,18]$ and odd-Fr [21] isotopes.

of the $25 / 2^{+}$and $23 / 2^{-}$states is only $450 \mathrm{keV}$ in ${ }^{211} \mathrm{Fr}$ [21]. In ${ }^{209} \mathrm{Fr}$, with two additional neutron holes, it is plausible that the $i_{13 / 2}$ orbit has come down in energy (or the $f_{7 / 2}$ orbit has gone up) such that the $25 / 2^{+}$energy is comparable to or even below that of the $23 / 2^{-}$state. In this case, rather than change configurations, the decay path should primarily populate the remaining $21 / 2^{+}, 17 / 2^{+}$, and $13 / 2^{+}$members of the $\pi i_{13 / 2} \otimes\left(\pi h_{9 / 2}\right)^{2}$ configuration. In addition to the excitation energies of the levels, we can compare the number of transitions in a cascade to the number expected for different configurations. For example, either the $\pi i_{13 / 2} \otimes \pi\left(h_{9 / 2}\right)^{2}$ or the $\pi f_{7 / 2} \otimes \pi\left(h_{9 / 2}\right)^{2}$ configuration allows four transitions, since the odd proton occupies a different orbital than the pair of coupled protons. However, if the odd proton occupies the same orbital as the pair of coupled protons $\pi h_{9 / 2} \otimes \pi\left(h_{9 / 2}\right)^{2}$, the odd proton Pauli blocks one of the spin states, and only three transitions are observed.

With this background, we now turn to a discussion of the level scheme of ${ }^{209} \mathrm{Fr}$, shown in Fig. 6. The cascade consisting of the 632,724 , and $359 \mathrm{keV}$ transitions, shown on the left of Fig. 6 , is consistent with $\pi h_{9 / 2} \otimes \pi\left(h_{9 / 2}\right)^{2}$ configuration. The excitation energy of these levels fits the systematics of the At nuclei very well, as seen in Fig. 9. We tentatively assign the levels at 632,1356 , and $1715 \mathrm{keV}$ as the members of the $\pi\left(h_{9 / 2}\right)^{3}$ multiplet.

The isomeric level at $826 \mathrm{keV}$ is fed by a cascade of four transitions of energies 568, 404, 468, and $344 \mathrm{keV}$ as expected for members of the $\pi i_{13 / 2} \otimes \pi\left(h_{9 / 2}\right)^{2}$ or $\pi f_{7 / 2} \otimes \pi\left(h_{9 / 2}\right)^{2}$ configuration. (The angular distribution results for the 568 and $404 \mathrm{keV}$ transitions are consistent with stretched $E 2$. The statistics are too poor to allow multipolarity assignments for the 468 and $344 \mathrm{keV}$ transitions.) However, the excitation energy of the isomeric level, $826 \mathrm{keV}$, is consistent with the energy of the known $13 / 2^{+}$state in neighboring ${ }^{205} \mathrm{At}$ and is significantly higher than the energy of the $f_{7 / 2}$ states 


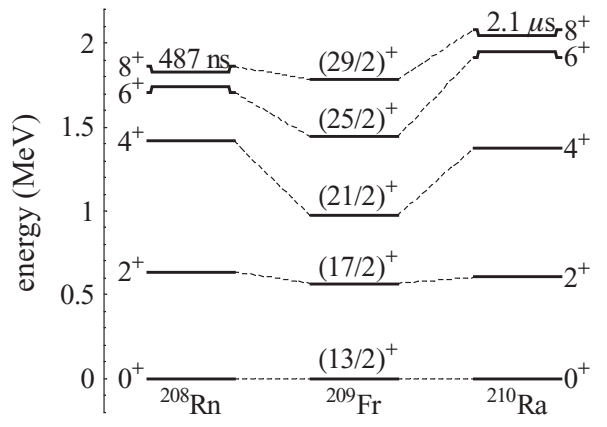

FIG. 10. Comparison of similar configurations in the $N=$ $122 \mathrm{Rn}[15], \mathrm{Fr}$, and $\mathrm{Ra}[36]$ isotopes.

in ${ }^{205,207,209}$ At ( $\left.~ 300-500 \mathrm{keV}\right)$. In addition, the conversion coefficient deduced for the $194 \mathrm{keV}$ transition is consistent with an $E 1$ assignment $\left(13 / 2^{+} \rightarrow 13 / 2^{-}\right)$, but definitely rules out an $M 3$ character $\left(7 / 2^{-} \rightarrow 13 / 2^{+}\right)$. Thus we assign spin and parity of $13 / 2^{+}$to the level at $826 \mathrm{keV}$ and the $\pi i_{13 / 2} \otimes \pi\left(h_{9 / 2}\right)^{2}$ configuration to this cascade.

The relative spacing of the $\pi i_{13 / 2} \otimes \pi\left(h_{9 / 2}\right)^{2}$ levels should be similar to those depopulating the $8^{+} \pi\left(h_{9 / 2}\right)^{2}$ isomers in ${ }^{208} \mathrm{Rn}$ and ${ }^{210} \mathrm{Ra}$, the neighboring $N=122$ nuclei. The level spacings are compared in Fig. 10 where it can be seen that they do indeed follow the same general pattern but with a more equal spacing in ${ }^{209} \mathrm{Fr}$. The alteration in this spacing could be an effect of the odd proton's interaction with the coupled $h_{9 / 2}$ proton pair.

When the structure of the nucleus is dominated by a relatively high- $j(j \geqslant 7 / 2)$ orbital, seniority, the number of unpaired particles, may be a good quantum number [30-34]. In this case, transitions can be classified as seniority changing or seniority conserving. The $B(E 2)$ values for these two classes of transitions follow very different trends as a function of the fractional filling of the single particle orbit: bowlshaped with the $B(E 2)$ minimizing in mid-shell for seniority conserving transitions and arch-shaped $[B(E 2)$ maximum in midshell] for seniority changing transitions [35]. As shown in Ref. [30], the even-even nuclei in the translead region (Po, $\mathrm{Rn}$, and $\mathrm{Ra}$ isotopes) are particularly good examples of the seniority framework. In the ${ }^{209} \mathrm{Fr}$ level scheme, the two cascades discussed above follow the expected pattern of transitions depopulating a seniority isomer. The 632, 724, and $359 \mathrm{keV}$ transitions are tentatively assigned the $\pi h_{9 / 2} \otimes$ $\pi\left(h_{9 / 2}\right)^{2}$ configuration, while the $568,404,468$, and $344 \mathrm{keV}$ cascade is tentatively assigned the $\pi i_{13 / 2} \otimes \pi\left(h_{9 / 2}\right)^{2}$ configuration. For both, the $h_{9 / 2}$ high- $j$ shell is filled to about mid shell, so that the $B(E 2)$ values for both the seniority conserving 359 and $344 \mathrm{keV}$ transitions are expected to be minimized. Assuming a generous upper limit of 1 W.u. for the $B(E 2)$ value, the lifetime of the maximally aligned seniority isomeric states, depopulated by the 359 and $344 \mathrm{keV}$ transitions, in ${ }^{209} \mathrm{Fr}$ is estimated to be less than a few ns. This is consistent with the generally observed shorter halflives as we move away from magicity and with the observed $2.1 \mathrm{~ns}$ half-life of the equivalent state in ${ }^{211} \mathrm{Fr}[21]$.

\section{CONCLUSION}

States in ${ }^{209} \mathrm{Fr}$ have been identified for the first time up to $2.6 \mathrm{MeV}$ in excitation energy. Both prompt and delayed gamma ray spectra were used to construct the level scheme and identify the configurations of excited states in ${ }^{209} \mathrm{Fr}$. A cascade of three transitions depopulated what is probably the $21 / 2^{-}$maximally aligned $\pi h_{9 / 2}^{3}$ state at $1715 \mathrm{keV}$ and feeds the $9 / 2^{-}$ground state. A configuration isomer, likely based on the $\pi i_{13 / 2}$ state with a half-life of $446 \mathrm{~ns}$ was observed at an excitation energy of $826 \mathrm{keV}$. Four transitions, with possible $\pi i_{13 / 2} \otimes \pi\left(h_{9 / 2}\right)^{2}$ configuration are found to feed into this isomeric level.

\section{ACKNOWLEDGMENTS}

We thank G. Hagemann for insightful discussion and calculations regarding the potential energy surface and the staff of the ESTU accelerator for their efforts. One of us (J.Y.Z.) thanks Dr. M. Schlett for making relevant codes available for theoretical calculations. Research has been supported by the U.S. DOE under Grant No. DE-FG02-91ER-40609, DE-FG02-05ER41379, DE-FG52-05NA25929, DE-FG0296ER40983, and DE-FG02-88ER40417.
[1] R. V. F. Janssens and T. L. Khoo, Annu. Rev. Nucl. Part. Sci. 41, 321 (1991).

[2] R. M. Clark, J. Phys. G: Nucl. Part. Phys. 25, 695 (1999).

[3] Balraj Singh, Roy Zywina, and Richard B. Firestone, Nucl. Data Sheets 97, 241 (2002).

[4] G. D. Dracoulis, A. P. Byrne, A. M. Baxter, P. M. Davidson, T. Kibedi, T. R. McGoram, R. A. Bark, and S. M. Mullins, Phys. Rev. C 60, 014303 (1999).

[5] J. F. C. Cocks et al., Eur. Phys. J. A 3, 17 (1998).

[6] T. Grahn et al., Eur. Phys. J. A 25, 441 (2005).

[7] M. R. Schmorak, Nucl. Data Sheets 75, 667 (1995).

[8] Shaheen Rab, Nucl. Data Sheets 71, 421 (1994).

[9] M. R. Schmorak, Nucl. Data Sheets 80, 647 (1997).
[10] F. G. Kondev, Nucl. Data Sheets 105, 1 (2005).

[11] M. R. Schmorak, Nucl. Data Sheets 72, 409 (1994).

[12] F. G. Kondev, Nucl. Data Sheets 101, 521 (2004).

[13] E. Browne, Nucl. Data Sheets 88, 29 (1999).

[14] M. J. Martin, Nucl. Data Sheets 70, 315 (1993).

[15] M. J. Martin, Nucl. Data Sheets 47, 797 (1986).

[16] M. J. Martin, Nucl. Data Sheets 63, 723 (1991).

[17] E. Browne, Nucl. Data Sheets 99, 649 (2003).

[18] E. Browne, Nucl. Data Sheets 103, 183 (2004).

[19] E. Browne, Nucl. Data Sheets 104, 427 (2005).

[20] Y. A. Akovali, Nucl. Data Sheets 66, 237 (1992).

[21] A. P. Byrne, G. D. Dracoulis, C. Fahlander, H. Hübel, A. R. Poletti, A. E. Stuchbery, J. Gerl, R. F. Davie, and S. J. Poletti, Nucl. Phys. 448, 137 (1986). 
[22] P. Kuusiniemi, F. P. Heßberger, D. Ackermann, S. Hofmann, and I. Kojouharov, Eur. Phys. J. A 23, 417 (2005).

[23] C. W. Beausang et al., Nucl. Instrum. Methods Phys. Res. A 452, 431 (2000).

[24] J. J. Ressler et al., Nucl. Instrum. Methods Phys. Res. B 204, 141 (2003).

[25] L. Corradi et al., Phys. Rev. C 71, 014609 (2005).

[26] J. F. Ziegler, J. P. Biersack, and U. Littmark, The Stopping and Range of Ions in Solids (Pergamon Press, New York, 1985).

[27] A. Winther and J. de Boer, A Computer Program for Multiple Coulomb Excitation, California Institute of Technology, Technical Report (November 18, 1965).
[28] C. W. Beausang et al., to be published.

[29] T. Bengtsson, Nucl. Phys. A496, 56 (1989).

[30] J. J. Ressler et al., Phys. Rev. C 69, 034317 (2004).

[31] G. Racah, Phys. Rev. 63, 367 (1943).

[32] A. K. Kerman, Ann. Phys. (NY) 12, 300 (1961).

[33] A. de Shalit and I. Talmi, Nuclear Shell Theory (Academic Press, New York, 1963).

[34] I. Talmi, Simple Models of Complex Nuclei (Harwood Academic Press, Switzerland, 1993).

[35] R. F. Casten, Nuclear Structure from a Simple Perspective (Oxford University Press, New York, 2000).

[36] J. J. Ressler et al., Phys. Rev. C 69, 034331 (2004). 